\title{
Odontogeriatras, geriatras e idosos brasileiros: uma análise por estados e regiões do país
}

\author{
Dental geriatricians, geriatricians and Brazilian elderly: an analysis by states \\ and regions of the country
}

\author{
Odontogeriatras, geriatras y ancianos brasileños: un análisis de los estados \\ y regiones del país
}

\author{
José de Alencar FERNANDES NETO ${ }^{1}$ \\ Andrea Maria Teles SILVA ${ }^{2}$ \\ Maria Helena Chaves de Vasconcelos CATÃ̃ ${ }^{3}$ \\ ${ }^{l}$ Mestrando do Programa de Pós-Graduação em Odontologia, Departamento de Odontologia, Universidade Estadual da Paraíba, \\ UEPB, 58429-500, Campina Grande - PB, Brasil \\ ${ }^{2}$ Cirurgiã-Dentista, Departamento de Odontologia, Universidade Estadual da Paraíba \\ UEPB, 58429-500, Campina Grande - PB, Brasil \\ ${ }^{3}$ Professora Doutora do Programa de Pós-Gradução em Odontologia, Universidade Estadual da Paraíba, \\ UEPB, 58429-500, Campina Grande - PB, Brasil
}

\begin{abstract}
Resumo
Introdução: A população idosa vem crescendo expressivamente ao longo dos anos no Brasil e com isso, nota-se uma maior procura por atendimentos especializados neste público. Objetivo: calcular e avaliar as proporções entre odontogeriatras, geriatras e idosos, de acordo com os estados e regiões do Brasil. Material e método: Buscou-se o número de profissionais em atividade segundo o Conselho Federal de Odontologia (CFO) e do Conselho Federal de Medicina (CFM) e o número de idosos através do Instituto Brasileiro de Geografia e Estatística (IBGE). Todos os dados utilizados nesta pesquisa são de acesso público. Resultados: Constatou-se que existem 274 odontogeriatras e 1112 geriatras no Brasil. A proporção odontogeriatra/geriatra no país é de 1/4,06 e no Sul essa relação é menor (1/2,72). O Sudeste abriga a maior parte dos odontogeriatras e geriatras, correspondendo a $54,02 \%$ e $51,08 \%$, respectivamente. No Sul observa-se o menor número de idosos por profissional, com 38.676,05 por odontogeriatra e 14.170,10 por geriatra. Conclusão: Com o pequeno número de profissionais somado com a grande quantidade de idosos, conclui-se que há uma escassez de odontogeriatras e geriatras em determinados estados e regiões do Brasil.
\end{abstract}

Descritores: Odontologia Geriátrica; Geriatria; Idoso; Serviços de Saúde para Idosos.

\begin{abstract}
Introduction: The elderly population has grown significantly over the years in Brazil and with this, it's noticed a greater demand for specialized care in this audience. Objective: to calculate and evaluate the proportions between dental geriatricians, geriatricians and the elderly people, according to the states and regions of Brazil. Material and method: We attempted to the number of registered professionals according to the Federal Council of Dentistry (CFO) and the Federal Council of Medicine (CFM) and the number of older people dated by the Brazilian Institute of Geography and Statistics (IBGE). All data used in this research are of public access. Results: It was found that there are 274 professionals in geriatric dentistry and 1,112 geriatricians in Brazil. The proportion dental geriatrician/geriatrician in the country is $1 / 4.06$ and in the South this ratio is smaller $(1 / 2.72)$. The Southeast is home to the most dental geriatricians and geriatricians, corresponding to $54.02 \%$ and $51.08 \%$, respectively. In the South, the fewest elderly per professional ratio is seen with 38,676.05 per dental geriatrician and 14170.10 per geriatrician. Conclusion: With the small number of professionals coupled with the large number of elderly, it is concluded that there is a shortage of geriatricians and dental geriatricians in certain states and regions of Brazil.
\end{abstract}

Descriptors: Geriatric Dentistry; Geriatrics; Aged; Health Services for the Aged.

\section{Resumen}

Introducción: La población adulta mayor ha crecido significativamente en los últimos años en Brasil y con esto, se nota una demanda mayor de atención especializada en este público. Objetivo: calcular y evaluar las proporciones entre odontogeriatras, geriatras y ancianos, de acuerdo con los estados y las regiones de Brasil. Materiales y métodos: Se buscó la información del número de profesionales en actividad registrados en el Consejo Federal de Odontología (CFO) y el Consejo Federal de Medicina (CFM) y el número de adultos mayores, a través del Instituto Brasileño de Geografía y Estadística (IBGE). Todos los datos utilizados en esta investigación son de acceso público. Resultados: Se encontró que existen 274 odontogeriatras y 1112 geriatras en Brasil. La proporción odontogeriatra/geriatra en el país es de 1/4,06 y en el sur del país esta relación es menor (1/2.72). El Sudeste tiene la mayor proporción de odontogeriatras y geriatras, correspondientes al 54,02\% y 51,08\%, respectivamente. En el sur se observa el menor número de adultos mayores por profesional con 38.676,05 por odontogeriatra y $14.170,10$ por geriatra. Conclusión: con el pequeño número de profesionales, sumado con la gran cantidad de adultos mayores, se concluye que existe una escasez de geriatras y odontogeriatras en algunos estados y regiones de Brasil.

Descriptores: Odontología Geriátrica; Geriatría; Anciano; Servicios de Salud para Ancianos. 


\section{INTRODUÇÃO}

A população idosa vem crescendo expressivamente ao longo dos anos, tanto em países desenvolvidos como em países em desenvolvimento. No Brasil, observa-se que este crescimento vem acontecendo de forma ainda mais acelerada e dentre os fatores que podem explicar este processo estão o aumento da expectativa de vida e a queda nas taxas de mortalidade da população brasileira ${ }^{1-4}$.

Com o aumento do número de idosos no país, nota-se consequentemente uma maior procura por atendimentos especializados neste público com atenções específicas, formas de avaliação e tratamentos diferenciados nas diversas áreas da saúde. Os profissionais, portanto, devem conhecer profundamente as características e peculiaridades dos idosos, assim como suas enfermidades, buscando caminhos que levem a um envelhecimento saudável, digno, ativo e a maior qualidade de vida ${ }^{5-8}$.

É natural no processo de envelhecimento o funcionamento mais lento do organismo, e com isso o surgimento de diversas limitações e doenças, tanto fisiológicas quanto psicológicas. Além disso, o indivíduo idoso pode apresentar alterações sistêmicas sendo importante orientações de profissionais de saúde para que haja conscientização e mudança de hábitos desses indivíduos ${ }^{6}$.

A Geriatria, introduzida no Brasil apenas na década de 70, é a especialidade médica responsável pelos aspectos clínicos do envelhecimento e pelos amplos cuidados de saúde necessários às pessoas idosas. É a área médica que cuida da saúde e das doenças da velhice com o principal objetivo de otimizar a qualidade de vida e a autonomia dos idosos. O geriatra, portanto, deve ter uma visão integral do idoso, principalmente daquele paciente portador de várias doenças 5,7 .

O aumento da população idosa no Brasil tem favorecido também o crescimento do mercado odontológico, pois se observa que idosos e/ou seus familiares têm buscado cirurgiões-dentistas com uma formação mais completa, capacitados ou especialistas para a atenção odontogeriátrica e que atuem de forma multiprofissional e multidisciplinar, visto que é notável a inter-relação entre a condição sistêmica e a oral ${ }^{4}$.

A odontologia voltada para a terceira idade, a Odontogeriatria, foi regulamentada no ano de 2001 pelo Conselho Federal de Odontologia (CFO), tornando-se uma especialidade que concentra o estudo dos fenômenos decorrentes do envelhecimento que também tem repercussão na boca e suas estruturas associadas, bem como a promoção de saúde, o diagnóstico, a prevenção e o tratamento de enfermidades bucais e do sistema estomatognático do idoso ${ }^{1,3,9}$.

Conhecendo a importância e necessidade de profissionais especialistas em saúde do idoso em todo o Brasil, o objetivo deste trabalho foi calcular e avaliar o número de odontogeriatras e geriatras em atividade no país e suas proporções quanto ao número de idosos, cirurgiões-dentistas e médicos, de acordo com os estados e regiões brasileiras.

\section{MATERIAL E MÉTODO}

Os dados referentes ao número total de profissionais no Brasil e por Unidades da Federação foram extraídos diretamente dos sites do $\mathrm{CFO}^{10} \mathrm{e}$ do Conselho Federal de Medicina - $\mathrm{CFM}^{11}$. Obtiveram-se o número total de profissionais em atividade no Brasil, especialistas em odontogeriatria e geriatria, cadastrados e em atividade, assim como a distribuição geográfica desses de acordo com as Unidades da Federação e regiões do país.

No site do Instituto Brasileiro de Geografia e Estatística - IBGE $^{12}$ foram coletadas as informações relativas à população idosa (indivíduos acima dos 60 anos de idade), tomando como base o Censo Demográfico 2010.

As pesquisas foram realizadas no dia 2 de março de 2016 no site do CFO e no dia 3 de março de 2016 nos sites do CFM, conseguindo-se assim o número exato de profissionais devidamente regulamentados nos Conselhos até as datas das buscas. Todos os dados utilizados nesta pesquisa são de acesso público.

\section{RESULTADOS}

Atualmente, existem 274 odontogeriatras e 1112 geriatras no Brasil, cadastrados no CFO e CFM, respectivamente. Os estados do Acre, Maranhão e Roraima ainda não possuem especialistas em Odontogeriatria e Rondônia é o único estado brasileiro que não possui geriatra. A relação entre odontogeriatra/geriatra encontrada no país foi de 1/4,06 (Tabela 1).

Por regiões brasileiras, verificou-se que o Sudeste abriga a maior parte dos odontogeriatras e geriatras, correspondendo a $54,02 \%$ e $51,08 \%$, respectivamente. No Sul, a relação entre o número de odontogeriatras por geriatras foi a menor $(1 / 2,72)$ com relação as outras regiões do país (Tabela 2 ).

Observou-se que no Brasil a cada 1016,03 cirurgiões-dentistas, 1 é especialista em Odontogeriatria $(1 / 1016,03)$ e a cada 337,77 médicos, 1 é geriatra $(1 / 337,77)$. Essas relações diminuem ou aumentam de acordo com os estados brasileiros (Tabela 3 ).

Santa Catarina é o estado brasileiro com o maior número de odontogeriatras por idosos $(1 / 26.276,52)$ e o Distrito Federal apresenta o maior número de médicos geriatras por indivíduos na terceira idade $(1 / 5.988,27)$. Excluindo os estados que não possuem odontogeriatras, verificou-se que os três estados com maior número de idosos por profissionais estão localizados na região Nordeste: Ceará, Bahia e Piauí (Tabela 4). 
Tabela 1. Número de odontogeriatras, geriatras e a relação entre a quantidade dos dois profissionais por Unidade da Federação de de acordo com os dados cadastrados no CFO e CFM

\begin{tabular}{lccc}
$\begin{array}{c}\text { Unidades da } \\
\text { Federação }\end{array}$ & $\begin{array}{c}\text { Número de } \\
\text { Odontogeriatras }\end{array}$ & $\begin{array}{c}\text { Número } \\
\text { de } \\
\text { Geriatras }\end{array}$ & $\begin{array}{c}\text { Relação Odontogeriatra/ } \\
\text { Geriatra }\end{array}$ \\
\hline Acre & 0 & 01 & $0 / 1$ \\
Alagoas & 02 & 20 & $1 / 10$ \\
Amapá & 01 & 02 & $1 / 2$ \\
Amazonas & 01 & 02 & $1 / 2$ \\
Bahia & 05 & 35 & $1 / 7$ \\
Ceará & 02 & 28 & $1 / 14$ \\
Distrito Federal & 04 & 33 & $1 / 8,25$ \\
Espírito Santo & 11 & 41 & $1 / 3,72$ \\
Goiás & 05 & 45 & $1 / 9$ \\
Maranhão & 0 & 08 & $0 / 8$ \\
Mato Grosso & 01 & 23 & $1 / 23$ \\
Mato Grosso do Sul & 02 & 07 & $1 / 3,5$ \\
Minas Gerais & 23 & 157 & $1 / 6,82$ \\
Pará & 04 & 16 & $1 / 4$ \\
Paraíba & 04 & 16 & $1 / 4$ \\
Paraná & 21 & 83 & $1 / 3,95$ \\
Pernambuco & 03 & 28 & $1 / 9,33$ \\
Piauí & 01 & 09 & $1 / 9$ \\
Rio de Janeiro & 45 & 89 & $1 / 1,97$ \\
Rio Grande do Norte & 02 & 12 & $1 / 6$ \\
Rio Grande do Sul & 39 & 93 & $1 / 2,38$ \\
Rondônia & 02 & 0 & $2 / 0$ \\
Roraima & 0 & 02 & $0 / 2$ \\
Santa Catarina & 25 & 56 & $1 / 2,24$ \\
São Paulo & 69 & 289 & $1 / 4,18$ \\
Sergipe & 01 & 11 & $1 / 11$ \\
Tocantins & 01 & 6 & $1 / 6$ \\
Total no Brasil & $\mathbf{2 7 4}$ & $\mathbf{1 1 1 2}$ & $\mathbf{1 / 4 , 0 6}$ \\
\hline & & &
\end{tabular}

Tabela 2. Número, distribuição percentual e relação de odontogeriatras e geriatras por região brasileira

\begin{tabular}{|c|c|c|c|c|c|}
\hline $\begin{array}{c}\text { Região do } \\
\text { Brasil }\end{array}$ & $\begin{array}{l}\mathbf{N}^{\mathbf{o}} \text { de } \\
\text { ODG }^{*}\end{array}$ & \% ODG & $\begin{array}{l}\mathbf{N}^{\mathbf{0}} \mathbf{d e} \\
\text { GER R }^{* *}\end{array}$ & \% GER & $\begin{array}{c}\text { Relação } \\
\text { ODG/GER }\end{array}$ \\
\hline Centro-Oeste & 12 & $4,38 \%$ & 108 & $9,71 \%$ & $1 / 9$ \\
\hline Nordeste & 20 & $7,30 \%$ & 167 & $15,02 \%$ & $1 / 8,35$ \\
\hline Norte & 09 & $3,29 \%$ & 29 & $2,61 \%$ & $1 / 3,22$ \\
\hline Sudeste & 148 & $54,02 \%$ & 576 & $51,80 \%$ & $1 / 3,89$ \\
\hline Sul & 85 & $31,01 \%$ & 232 & $20,86 \%$ & $1 / 2,72$ \\
\hline Total & 274 & $100,00 \%$ & 1112 & $100,00 \%$ & $1 / 4,06$ \\
\hline
\end{tabular}

*ODG - Odontogeriatras, **GER - Geriatras

Tabela 3. Relação entre odontogeriatras/cirurgiões-dentistas e geriatras/médicos nas Unidades Federativas Brasileiras

\begin{tabular}{|c|c|c|c|c|}
\hline $\mathbf{U F}$ & $\begin{array}{c}\mathbf{N}^{\circ} \\
\operatorname{de}^{\text {CD }}{ }^{*}\end{array}$ & $\begin{array}{c}\text { Relação } \\
\text { ODG }^{* *} / \text { CD }\end{array}$ & $\begin{array}{c}N^{\mathbf{o}} \mathbf{d e} \\
\text { MÉD }^{* * * *}\end{array}$ & $\begin{array}{c}\text { Relação } \\
\mathbf{G E R}^{* * * * *} / \mathbf{M E ́ D}\end{array}$ \\
\hline Acre & 624 & $0 / 624$ & 768 & $1 / 768$ \\
\hline Alagoas & 2.666 & $1 / 1.333$ & 4.211 & $1 / 210,55$ \\
\hline Amapá & 610 & $1 / 610$ & 628 & $1 / 314$ \\
\hline Amazonas & 3.586 & $1 / 3.586$ & 4.505 & $1 / 2.252,5$ \\
\hline Bahia & 11.035 & $1 / 2.207$ & 18.924 & $1 / 540,68$ \\
\hline Ceará & 6.191 & $1 / 3.095,50$ & 11.675 & $1 / 416,96$ \\
\hline $\begin{array}{l}\text { Distrito } \\
\text { Federal }\end{array}$ & 6.906 & $1 / 1,726,50$ & 11.317 & $1 / 342,93$ \\
\hline Espírito Santo & 5.356 & $1 / 486,90$ & 8.696 & $1 / 212,09$ \\
\hline Goiás & 9.565 & $1 / 1.913$ & 11.055 & $1 / 245,66$ \\
\hline Maranhão & 3.551 & $0 / 3.551$ & 4.679 & $1 / 584,87$ \\
\hline Mato Grosso & 4.152 & 1/4.152 & 4.395 & $1 / 191,08$ \\
\hline $\begin{array}{l}\text { Mato Grosso } \\
\text { do Sul }\end{array}$ & 3.764 & $1 / 1882$ & 4.624 & $1 / 660,57$ \\
\hline Minas Gerais & 32.689 & $1 / 1.421$ & 45.371 & $1 / 288,98$ \\
\hline Pará & 4.760 & $1 / 1.190$ & 7.185 & $1 / 449,06$ \\
\hline Paraíba & 4.076 & $1 / 1.019$ & 5.777 & $1 / 361,06$ \\
\hline Paraná & 17.549 & $1 / 835,66$ & 21.746 & $1 / 262$ \\
\hline Pernambuco & 7.543 & $1 / 2.514,33$ & 14.708 & $1 / 525,28$ \\
\hline Piauí & 2.682 & 1/2.682 & 3.843 & $1 / 427$ \\
\hline Rio de Janeiro & 29.788 & $1 / 661,95$ & 61.685 & $1 / 693,08$ \\
\hline $\begin{array}{l}\text { Rio Grande do } \\
\text { Norte }\end{array}$ & 3.487 & 1/1743,50 & 5.024 & $1 / 418,66$ \\
\hline $\begin{array}{c}\text { Rio Grande do } \\
\text { Sul }\end{array}$ & 17.255 & $1 / 442,43$ & 28.545 & $1 / 306,93$ \\
\hline Rondônia & 1.964 & 1/982 & 2.195 & 0/2.195 \\
\hline Roraima & 599 & $0 / 599$ & 673 & $1 / 336,6$ \\
\hline $\begin{array}{c}\text { Santa } \\
\text { Catarina }\end{array}$ & 11.002 & $1 / 440,08$ & 13.060 & $1 / 233,21$ \\
\hline São Paulo & 83.265 & $1 / 1.206,73$ & 119.472 & $1 / 413,39$ \\
\hline Sergipe & 1.838 & 1/1.838 & 3.296 & $1 / 299,63$ \\
\hline Tocantins & 1.891 & 1/1.891 & 2.025 & $1 / 337,5$ \\
\hline $\begin{array}{c}\text { Total no } \\
\text { Brasil }\end{array}$ & 278.394 & $1 / 1.016,03$ & 420.082 & $1 / 337,77$ \\
\hline
\end{tabular}

*CD- Cirurgiões-Dentistas; **ODG - Odontogeriatras; ***O total de médicos em atividade no país é o resultado do somatório das inscrições principais ativas em todas as Unidades da Federação, $* * * * \mathrm{GER}-$ Geriatras
Tabela 4. Relação entre a quantidade de odontogeriatras e geriatras por número de idosos em cada Unidade Federativa Brasileira

\begin{tabular}{|c|c|c|c|}
\hline UF & $\begin{array}{l}\text { Número de } \\
\text { Idosos }\end{array}$ & $\begin{array}{c}\text { Relação } \\
\text { Odontogeriatras/ } \\
\text { Idosos }\end{array}$ & $\begin{array}{c}\text { Relação } \\
\text { Geriatras/Idosos }\end{array}$ \\
\hline Acre & 46.926 & $0 / 46.926$ & $1 / 46.926$ \\
\hline Alagoas & 276.763 & $1 / 138.381,50$ & $1 / 13.838,15$ \\
\hline Amapá & 34.276 & $1 / 34.276$ & $1 / 17.138$ \\
\hline Amazonas & 210.225 & $1 / 210.225$ & $1 / 105.112,50$ \\
\hline Bahia & 1.451 .206 & $1 / 290.241,20$ & $1 / 41.463,02$ \\
\hline Ceará & 909.475 & $1 / 454.737,50$ & $1 / 32.481,25$ \\
\hline Distrito Federal & 197.613 & $1 / 49.403,25$ & $1 / 5.988,27$ \\
\hline Espírito Santo & 364.745 & $1 / 33.158,63$ & $1 / 8.896,21$ \\
\hline Goiás & 561.625 & 1/112.325 & $1 / 12.480,55$ \\
\hline Maranhão & 568.680 & $0 / 568.680$ & $1 / 71.085$ \\
\hline Mato Grosso & 239.626 & $1 / 239.626$ & $1 / 10.418,52$ \\
\hline $\begin{array}{l}\text { Mato Grosso do } \\
\text { Sul }\end{array}$ & 239.270 & $1 / 119.635$ & $1 / 34.181,42$ \\
\hline Minas Gerais & 2.310 .564 & $1 / 100.459,30$ & $1 / 14.716,96$ \\
\hline Pará & 535.134 & $1 / 133.783,50$ & $1 / 33.445,87$ \\
\hline Paraíba & 451.386 & $1 / 112.846,50$ & $1 / 28.211,62$ \\
\hline Paraná & 1.170 .955 & $1 / 55.759,76$ & $1 / 14.107,89$ \\
\hline Pernambuco & 937.943 & $1 / 312.647,66$ & $1 / 33.497,96$ \\
\hline Piauí & 330.949 & $1 / 330.949$ & $1 / 36.772,11$ \\
\hline Rio de Janeiro & 2.080 .608 & $1 / 46.235,73$ & 1/23.377,61 \\
\hline $\begin{array}{l}\text { Rio Grande do } \\
\text { Norte }\end{array}$ & 342.890 & $1 / 171.445$ & $1 / 28.574,16$ \\
\hline $\begin{array}{l}\text { Rio Grande do } \\
\text { Sul }\end{array}$ & 1.459 .597 & $1 / 37.425,56$ & $1 / 15.694,59$ \\
\hline Rondônia & 112.685 & $1 / 56.342,50$ & $0 / 112.685$ \\
\hline Roraima & 24.668 & 0/24.688 & $1 / 12.334$ \\
\hline Santa Catarina & 656.913 & $1 / 26.276,52$ & $1 / 11.730,58$ \\
\hline São Paulo & 4.771 .436 & $1 / 69.151,24$ & $1 / 16.510,15$ \\
\hline Sergipe & 185.957 & 1/185.957 & $1 / 16.905,18$ \\
\hline Tocantins & 117.554 & $1 / 117.554$ & $1 / 19.592,33$ \\
\hline Total no Brasil & 20.589 .669 & 1/75.144,77 & $1 / 18.515,88$ \\
\hline
\end{tabular}

As regiões Nordeste e Norte apresentam maior número de idosos por profissional. No Nordeste para cada odontogeriatra, existem aproximadamente 272.762 idosos e no Norte para cada geriatra, 37.292 indivíduos da terceira idade. A região Sul apresenta o menor número de idosos por profissional (Tabela 5).

Tabela 5. Relação entre a quantidade de odontogeriatras e geriatras por número de idosos nas regiões do Brasil

\begin{tabular}{lccc}
\hline $\begin{array}{c}\text { Região do } \\
\text { Brasil }\end{array}$ & $\begin{array}{c}\text { No de } \\
\text { idosos }\end{array}$ & $\begin{array}{c}\text { Relação } \\
\text { Odontogeriatras/ Idosos }\end{array}$ & $\begin{array}{c}\text { Relação } \\
\text { Geriatras/ Idosos }\end{array}$ \\
\hline Centro-Oeste & 1.238 .134 & $1 / 103.177,83$ & $1 / 11.464,20$ \\
Nordeste & 5.455 .249 & $1 / 272.762,45$ & $1 / 32.666,16$ \\
Norte & 1.081 .468 & $1 / 120.163,11$ & $1 / 37.292$ \\
Sudeste & 9.527 .353 & $1 / 64.374$ & $1 / 16.540,54$ \\
Sul & 3.287 .465 & $1 / 38.676,05$ & $1 / 14.170,10$ \\
Total & $\mathbf{2 0 . 5 8 9 . 6 6 9}$ & $\mathbf{1 / 7 5 . 1 4 4 , 7 7}$ & $\mathbf{1 / 1 8 . 5 1 5 , 8 8}$ \\
\hline
\end{tabular}

\section{DISCUSSÃO}

Com o aumento da expectativa de vida da população, é necessária uma melhora na qualidade de vida, e para isso a saúde desses indivíduos precisa ser subsidiada por profissionais capacitados para diagnosticar e tratar doenças específicas dessa idade. $\mathrm{Na}$ saúde sistêmica o profissional capacitado chama-se geriatra e na saúde bucal, odontogeriatra, os quais precisam trabalhar em conjunto, atentando que o paciente é um sistema complexo e que muitas alterações sistêmicas apresentam manifestações bucais. Assim sendo, na tentativa de melhorar a qualidade de vida desses, cabe um atendimento por equipe, utilizando uma abordagem interdisciplinar. Portanto, geriatras e odontogeriatras devem buscar soluções conjuntas ${ }^{1,4,6,13}$.

A odontogeriatria é uma especialidade que só foi regulamentada pelo Conselho Federal de Odontologia (CFO) no ano de 2001, ou seja, é relativamente nova, o que justifica o menor número de profissionais. Além 
disso, as faculdades de Odontologia no país não possuem em seu currículo, como disciplina obrigatória, a odontogeriatria, pois a disciplina não faz parte do currículo mínimo instituído pelo Ministério da Educação (MEC). Portanto, como a disciplina não foi estudada, suscita um menor interesse por parte dos alunos, gerando uma menor procura ao término do curso por uma especialização nesta área, já que os alunos não são informados da importância, mercado e lucro desta especialidade $\mathrm{e}^{10,14}$.

Considerando as alterações fisiológicas e psicológicas que acometem os idosos é necessário que os profissionais envolvidos no atendimento desses pacientes entendam que muitas alterações sistêmicas apresentam manifestações orais e na tentativa de melhorar a qualidade de vida destes cabe um atendimento por uma equipe utilizando uma abordagem interdisciplinar ${ }^{6}$.

Com base na pesquisa realizada, observamos um número reduzido de profissionais em todo o país, especialmente nas regiões norte e nordeste. Isto pode ser explicado pela inferior qualidade de vida nesta região, devido ao baixo desenvolvimento social e econômico da população, menor repasse de dinheiro público e investimento nestas regiões, fazendo com que a infraestrutura e equipamentos sejam precários e/ou escassos, tornando as condições de trabalho difíceis, levando esses profissionais a migrarem para outras regiões. Outro fator importante é a pouca valorização do profissional. Vendo estes que não há possibilidade de evolução em sua carreira, encontrando-se estagnados, migram para regiões com maiores e melhores oportunidades $^{15,16}$.

A pequena quantidade de odontogeriatras, somada com o aumento expressivo do número de idosos no país, faz com que a especialidade se torne uma área com promissor mercado de trabalho para a classe odontológica. Deve-se também considerar que grande parte dos especialistas, tanto na Odontologia como na Medicina, pode estar concentrada apenas em uma região específica dentro do estado, como em capitais ou cidades de médio e grande porte, gerando uma distribuição geográfica desigual de especialistas.

O aumento do número de escolas que ofereçam especializações em Odontogeriatria e Geriatria em todo o país e uma maior conscientização dos profissionais e da população da importância desta área seriam boas alternativas para impulsionar o aumento da quantidade de profissionais capacitados para o atendimento especializado ao público da terceira idade.

\section{CONCLUSÃO}

Com o envelhecimento da população brasileira se faz necessário o aumento de cirurgiões-dentistas e médicos especializados, que reconheçam e entendam as alterações especificas que ocorrem no organismo do idoso.
O pequeno número de profissionais somado com a grande quantidade de idosos brasileiros revela que em determinadas regiões e estados do Brasil há uma escassez e uma grande necessidade de profissionais especialistas em saúde do idoso, especialmente na área odontológica, tornando as duas especialidades, áreas de atuação com um mercado de trabalho promissor e de grande importância para a população.

\section{REFERÊNCIAS}

1. Rosa LB, Zuccolotto MCC, Bataglion C, Coronatto EAS. Odontogeriatria- a saúde bucal na terceira idade. RFO. 2008; 13(2):82-6.

2. Custodio W, Meloto CB, Gomes SGF, Barbosa CMR. Considerações clínicas em odontogeriatria: plano de tratamento integrado. Rev Bahiana Odonto. 2010; 1(1):19-26.

3. Ribeiro AFL, Leal MCC, Marques APO. Importância da Odontogeriatria no aspecto nutricional do idoso. RGO. 2012; 60(2): 241-6.

4. Irineu KN, Acioli Filho JAM, Costa RO, Catão MHCV. Saúde do idoso e o papel do odontólogo: inter-relação entre a condição sistêmica e a saúde bucal. Rev da FOL. 2015; 25(2):41-6.

5. Pereira AMVB, Schneider RH, Schwanke CHA. Geriatria, uma especialidade centenária. Sci Med. 2009; 19(4):154-61.

6. Araújo IDT, Freitas IN, Silva RB, Vasconcelos MG, Vasconcelos RG. Odontologia e abordagem interdisciplinar na atenção integral ao idoso relacionado às principais alterações orais. Com Ciencias Saúde. 2012; 23(1):81-92.

7. Kikuchi EL. Especialidades Médicas-Geriatria. Rev Med (São Paulo). 2012; 91(ed.esp.):33-7.

8. Santos MBF, Luthi LF, Zampieri MH, Consani RLX, Rizzatti-Barbosa CM. Tratamento endodôntico na terceira idade. RGO. 2013; 61:485-9.

9. Ribeiro DG, Silva MM, Nogueira SS, Arioli Filho JN. A saúde bucal na terceira idade. Salusvita. 2009; 28(1):101-11.

10. CFO: Conselho Federal de Odontologia. [Internet]. Citado em 2016 mar 02. Disponível em: http://cfo.org.br/

11. CFM: Conselho Federal de Medicina [Internet]. Portal Médico; 2010-2016. Citado em 2016 mar 3. Disponível em: http://portal.cfm.org.br/

12. IBGE: Instituto Brasileiro de Geografia e Estatística [Internet]. 2010. Citado em 2016 mar 03. Disponível em: http://www.ibge.gov.br/home/

13. Brunetti RF, Montenegro FLB. Odontogeriatria uma nova opção de trabalho no Século XXI. In: Cardoso $\mathrm{J}^{\mathrm{a}}$, Machado MEL. Odontologia: arte e conhecimento. São Paulo: Artes Médicas; 2003. p.440-453.

14. Ministério da Educação. Disponível em: http://portal.mec.gov.br/index.php 
15. Sousa TRV, Leite Filho PAM. Análise por dados em painel do status de saúde no Nordeste Brasileiro. Rev de Saúde Públ. 2008; 42(5): 796-804.

16. Silvestre JAC, de Aguiar ASW, Teixeira EH. Do Brasil sem dentes ao Brasil sorridente: um resgate histórico das políticas públicas de saúde bucal no Brasil. Cadernos ESP. 2013; 7(2):28-39.

\section{CONFLITO DE INTERESSES}

Os autores declaram não haver conflitos de interesse.

\section{AUTOR PARA CORRESPONDÊNCIA}

José de Alencar Fernandes Neto jneto411@hotmail.com

Submetido em 16/08/2016 Aceito em 19/09/2016 